\title{
Projective Chaos Synchronization Between the Different Chaotic Systems
}

\author{
Juan Bao, Gang Li, Jia Hou \\ College of Physics and Electronic Technology, \\ Liaoning Normal University \\ Dalian 116029, China
}

\begin{abstract}
The projective synchronization between the different chaotic systems is investigated based on the Lyapunov stability theory. The nonlinear feedback controllers are designed through constructing a Lyapunov function. The chaotic systems are synchronized by adjusting the feedback gain coefficients. This method can be applied for identical systems. Numerical simulations show that the synchronization method works well.
\end{abstract}

Keywords-Projective synchronization, Chaos, Lyapunov function, Nonlinear feedback

\section{INTRODUCTION}

Since the projective synchronization method was initially proposed by Mainieri and Rehacek[1], much attention has been devoted to research on projective synchronization of chaos due to its potential applications, and many synchronization methods have been proposed, such as, a modified projective synchronization(MPS) method is proposed and a general kind of proportional relationship between the drive system and response system is obtained[24]. An adaptive control scheme for the modified projective synchronization(AMPS) with uncertain parameters is presented[5-7,9]. The function projective synchronization(FPS) method is proposed to synchronize two chaotic systems based on the Lyapunov stability theory[ $8,10,11]$.

In this paper, the projective synchronization between the different chaotic systems and between two identical systems are investigated based on the Lyapunov stability theory. The nonlinear feedback controllers are designed through constructing a Lyapunov function. The two different chaotic systems are synchronized by adjusting the feedback gain coefficients. Numerical simulations show that the synchronization method works well.

\section{ProJeCtIVE SyNCHRONIZATION OF DifFERENT CHAOTIC SYSTEMS}

Rössler system is taken as the drive system, which is described as follow:

$$
\begin{aligned}
& \dot{x}_{1}=-\left(y_{1}+z_{1}\right) \\
& \dot{y}_{1}=x_{1}+a y_{1} \\
& \dot{z}_{1}=x_{1} z_{1}-c z_{1}+b
\end{aligned}
$$

\author{
Jingyun Jiang, Jia Li \\ College of Physics and Electronic Technology, \\ Liaoning Normal University \\ Dalian 116029, China
}

$$
\left\{\begin{array}{l}
\dot{x}_{2}=a_{1}\left(y_{2}-x_{2}\right)+u_{1} \\
\dot{y}_{2}=b_{1} x_{2}-x_{2} z_{2}-y_{2}+u_{2} \\
\dot{z}_{2}=x_{2} y_{2}-c_{1} z_{2}+u_{3}
\end{array}\right.
$$

where ${ }^{x_{1}}, y_{1},{ }_{1}$ and $x_{2}, y_{2},{ }_{2}$ are the state variables of the two systems. Rössler system and Lorenz system exhibit chaotic behaviour when system parameters are given by $a=b=0.2, c=5.7$ and $a_{1}=10, b_{1}=28, c_{1}=8 / 3$ respectively. The $u_{i}(i=1,2,3)$ are the nonlinear feedback controllers to be designed such that two chaotic systems can be synchronized in the following sense:

$$
\left\{\begin{array}{l}
\lim _{t \rightarrow \infty}\left\|x_{2}-\alpha x_{1}\right\|=0, \\
\lim _{t \rightarrow \infty}\left\|y_{2}-\beta y_{1}\right\|=0, \\
\lim _{t \rightarrow \infty}\left\|z_{2}-\gamma z_{1}\right\|=0 .
\end{array}\right.
$$

Without the controllers $u_{i}(i=1,2,3)$, the trajectories of the two different systems will quickly separate each other independently. But, the trajectories of the two controlled systems will approach synchronization in the above sense for any different initial condition by adjusting appropriate gain coefficients.

Theorem. For given scaling factors $\alpha, \beta, \gamma$, the propective synchronization between Rössler system and Lorenz system will be achieved by following controllers and the domain of the appropriate gain coefficients $\mathrm{ki}(i=1,2,3)$.

$$
\left\{\begin{array}{l}
u_{1}=f_{1}\left(x_{1}, y_{1}, z_{1}\right)-k_{1} e_{1} \\
u_{2}=f_{2}\left(x_{1}, y_{1}, z_{1}\right)-k_{2} e_{2} \\
u_{3}=f_{3}\left(x_{1}, y_{1}, z_{1}\right)-k_{3} e_{3}
\end{array}\right.
$$

where $f_{i}\left(x_{1}, y_{1}, z_{1}\right) \quad(i=1,2,3)$ are the functions of the state variables in drive system as follows:

$$
\left\{\begin{array}{l}
f_{1}\left(x_{1}, y_{1}, z_{1}\right)=\alpha a_{1} x_{1}-\left(\alpha+\beta a_{1}\right) y_{1}-\alpha z_{1} \\
f_{2}\left(x_{1}, y_{1}, z_{1}\right)=\left(\beta-\alpha b_{1}\right) x_{1}+(1+a) \beta y_{1}+\alpha \gamma x_{1} z_{1} \\
f_{3}\left(x_{1}, y_{1}, z_{1}\right)=-\alpha \beta x_{1} y_{1}-\left(c-c_{1}\right) \gamma z_{1}+\gamma x_{1} z_{1}+\gamma b
\end{array}\right.
$$

The domain of the feedback gain coefficients is given by

and Lorenz system as the response system is given by 


$$
\left\{\begin{array}{l}
k_{1} \geq-a_{1} \\
k_{2} \geq \frac{\left(a_{1}+b_{1}-\gamma z_{1}\right)^{2}}{2\left(a_{1}+k_{1}\right)}-1 \\
k_{3} \geq \frac{\beta^{2} y_{1}{ }^{2}}{2\left(a_{1}+k_{1}\right)}-c_{1}
\end{array}\right.
$$

Proof.Define

$e_{1}=x_{2}-\alpha x_{1}, e_{2}=y_{2}-\beta y_{1}, e_{3}=z_{2}-z_{1}$, then, the error dynamical system is given by

$$
\left\{\begin{array}{l}
\dot{e}_{1}=\dot{x}_{2}-\alpha \dot{x}_{1} \\
\dot{e}_{2}=\dot{y}_{2}-\beta \dot{y}_{1} \\
\dot{e}_{3}=\dot{z}_{2}-\gamma \dot{z}_{1}
\end{array}\right.
$$

The Lyapunov function is constructed as :

$$
V=\frac{1}{2}\left(e_{1}^{2}+e_{2}^{2}+e_{3}^{2}\right)
$$

The differential of the Lyapunov function along the trajectory of error dynamical system Eq.(7) is

$$
\begin{aligned}
\dot{V} & =e_{1} \dot{e}_{1}+e_{2} \dot{e}_{2}+e_{3} \dot{e}_{3} \\
& =e_{1}\left[-a_{1} e_{1}+a_{1} e_{2}+o a_{1} x_{1}+\left(\beta a_{1}+\alpha\right) y_{1}+\alpha z_{1}+u_{1}\right] \\
& +e_{2}\left[\left(b_{1}-\gamma_{1}\right) e_{1}-e_{2}-\alpha x_{1} e_{3}-e_{1} e_{3}+\left(o b_{1}-\beta\right) x_{1}-(1+a) \beta y_{1}-\alpha x_{1} z_{1}+u_{2}\right] \\
& +e_{3}\left[e_{1} e_{2}+\beta y_{1} e_{1}+\alpha x_{1} e_{2}-c_{1} e_{3}+\alpha \beta \beta_{1} y_{1}+\left(c-c_{1}\right) z_{1}-\not b-\gamma x_{1} z_{1}+u_{3}\right]
\end{aligned}
$$

considering Eq.(4), (5) and (6), we can obtain the derivative form of as follows:

$$
\begin{aligned}
& \dot{V}=-\frac{a_{1}+k_{1}}{2}\left(e_{1}-\frac{a_{1}+b_{1}-\gamma_{1}}{a_{1}+k_{1}} e_{2}\right)^{2}-\frac{a_{1}+k_{1}}{2}\left(e_{1}-\frac{\beta y_{1}}{a_{1}+k_{1}} e_{3}\right)^{2} \\
& -\left(1+k_{2}-\frac{\left(a_{1}+b_{1}-\gamma_{1}\right)^{2}}{2\left(a_{1}+k_{1}\right)}\right) e_{2}^{2}-\left(c_{1}+k_{3}-\frac{\beta^{2} y_{1}^{2}}{2\left(a_{1}+k_{1}\right)}\right) e_{3}^{2}
\end{aligned}
$$

Therefore, due to that a chaotic system is bounded, we can chose a set of appropriate feedback gain coefficients ki in Eq.(6) makes $\dot{V}<0$. The error states variables $e_{i}$ will become zero based on the Lyapunov stability theory. The projective synchronization is achieved.

\section{ProJeCtive SyNCHRONIZATION Of IDENTICAL CHAOTIC SYSTEMS}

By same method, the projective chaotic synchronization can be achieved between two identical systems also. Here the Rössler system is handled as the example. The drive system and response system are described as follow:

$$
\begin{aligned}
& \left\{\begin{array}{l}
\dot{x}_{2}=-\left(y_{2}+z_{2}\right) \\
\dot{y}_{2}=x_{2}+a y_{2} \\
\dot{z}_{2}=x_{2} z_{2}-c z_{2}+b
\end{array}\right. \\
& \left\{\begin{array}{l}
\dot{x}_{2}=-\left(y_{2}+z_{2}\right)+u_{1} \\
\dot{y}_{2}=x_{2}+a y_{2}+u_{2} \\
\dot{z}_{2}=x_{2} z_{2}-c z_{2}+b+u_{3}
\end{array}\right.
\end{aligned}
$$

where $u_{1}, u_{2}, u_{3}$ is nonlinear controllers. The error vectors are defined as $\left\{\begin{array}{l}e_{1}=x_{2}-\alpha x_{1} \\ e_{2}=y_{2}-\beta y_{1} \\ e_{3}=z_{2}-\gamma z_{1}\end{array}\right.$

Where the $\alpha, \beta, \gamma$ are the scaling factors. Then the following Lyapunov function is chosen

$V=\frac{1}{2}\left(e_{1}^{2}+e_{2}^{2}+e_{3}^{2}\right)$

We can take the controllers as

$\left\{\begin{array}{l}u_{1}=f_{1}\left(x_{1}, y_{1}, z_{1}\right)-k_{1} e_{1} \\ u_{2}=f_{2}\left(x_{1}, y_{1}, z_{1}\right)-k_{2} e_{2} \\ u_{3}=f_{3}\left(x_{1}, y_{1}, z_{1}\right)-k_{3} e_{3}\end{array}\right.$

$\left\{\begin{array}{l}f_{1}\left(x_{1}, y_{1}, z_{1}\right)=(\beta-\alpha) y_{1}+(\gamma-\alpha) z_{1} \\ f_{2}\left(x_{1}, y_{1}, z_{1}\right)=(\beta-\alpha) x_{1} \\ f_{3}\left(x_{1}, y_{1}, z_{1}\right)=(1-\alpha) \gamma x_{1} z_{1}+(\gamma-1) b\end{array}\right.$

Where the $f_{i}$ 's are the function of the state variables And the $k_{1}, k_{2}, k_{3}$ are the feedback gain coefficients.

Therefore, we can obtain the time derivation of the Lyapunov function along the state trajectory

$\dot{V}=e_{1}\left[-e_{2}-e_{3}+(\alpha-\beta) y_{1}+(\alpha-\gamma) z_{1}+u_{1}\right]$

$+e_{2}\left[e_{1}+a e_{2}+(\alpha-\beta) x_{1}+u_{2}\right]$

$+e_{3}\left[e_{1} e_{3}+\gamma z_{1} e_{1}+\left(\alpha x_{1}-c\right) e_{3}+(\alpha-1) \gamma x_{1} z_{1}+(1-\gamma) b+u_{3}\right]$

$=e_{1}\left[-k_{1} e_{1}-e_{2}-e_{3}\right]+e_{2}\left[e_{1}-\left(k_{2}-a\right) e_{2}\right]+e_{3}\left[\gamma z_{1} e_{1}-\left(k_{3}+c-\alpha x_{1}\right) e_{3}\right]$

$=-k_{1} e_{1}^{2}-\left(k_{2}-a\right) e_{2}^{2}-\left(k_{3}+c-\alpha x_{1}\right) e_{3}^{2}+\left(\gamma z_{1}-1\right) e_{1} e_{3}$

$=-\frac{k_{1}}{2} e_{1}^{2}-\left(k_{2}-a\right) e_{2}^{2}-\left(k_{3}+c-\alpha x_{1}-\frac{\left(\gamma z_{1}-1\right)^{2}}{2 k_{1}}\right) e_{3}^{2}$

$-\frac{k_{1}}{2}\left(e_{1}-\frac{\gamma z_{1}-1}{k_{1}} e_{3}\right)^{2}$

For $\dot{V} \leq 0$, the domain of those feedback factors $k_{i}$ are obtained

$\left\{\begin{array}{l}k_{1} \geq 0 \\ k_{2} \geq a \\ k_{3} \geq \alpha x_{1}-c+\frac{\left(\gamma z_{1}-1\right)^{2}}{2 k_{1}}\end{array}\right.$

\section{NUMERICAL SIMULATIONS}

In this section, the numerical examples is given to verify and illustrate the effectiveness of the proposed method.

Firstly, for the chaotic synchronization between the different systems, the domain of the ${ }^{y_{1}}$ and $z_{1}$ in the drive system are computed from the attractor of Lorenz system, which are $-10<x_{1}<13,-12<y_{1}<8$ and $0<z_{1}<24$ 
shown in Fig. 1, Fig. 2 and Fig.3. Selecting $k_{1}=20$, the $k_{2} \geq 23.1$ and $k_{3} \geq-2.2$ are attained, we take $k_{2}=28$ and $k_{3}=3$ here. During the simulation, the initial values of the drive and response systems are taken as $x_{1}(0)=3$ $y_{1}(0)=-5, z_{1}(0)=3$ and $x_{2}(0)=-4, y_{2}(0)=5$, $z_{2}(0)=-3$, the scaling factors are given by $\alpha=2, \beta=0.5, \gamma=3$, the system parameters are chosen as $a=0.2 \quad, \quad b=0.2 \quad, \quad c=5.7 \quad$ and $\quad a_{1}=10$ $b_{1}=28, c_{1}=8 / 3$. So the simulation results between the different systems are shown in Figure 1-Figure 4.

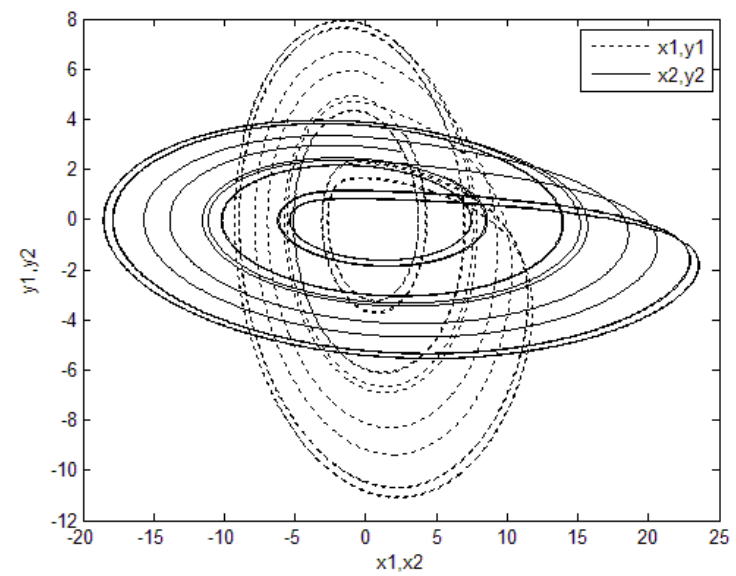

Figure 1. The attractors in projective synchronization for different systems.(1)

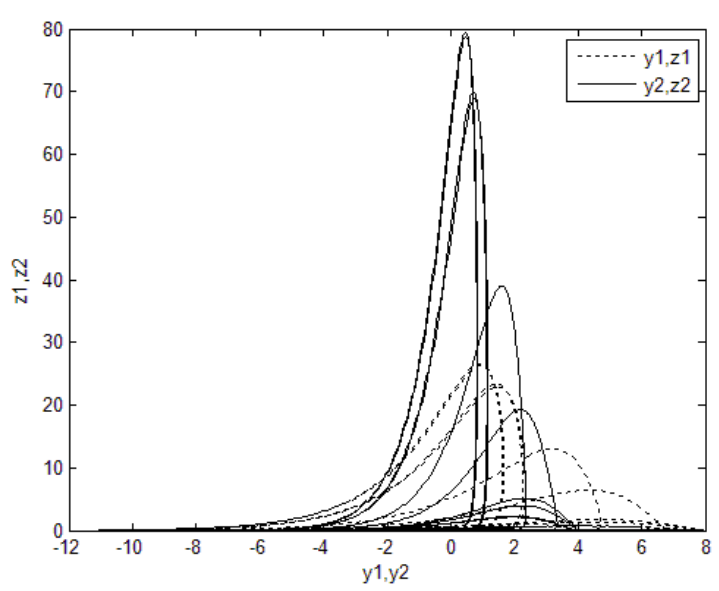

Figure 2. The attractors in projective synchronization for different systems.(2)

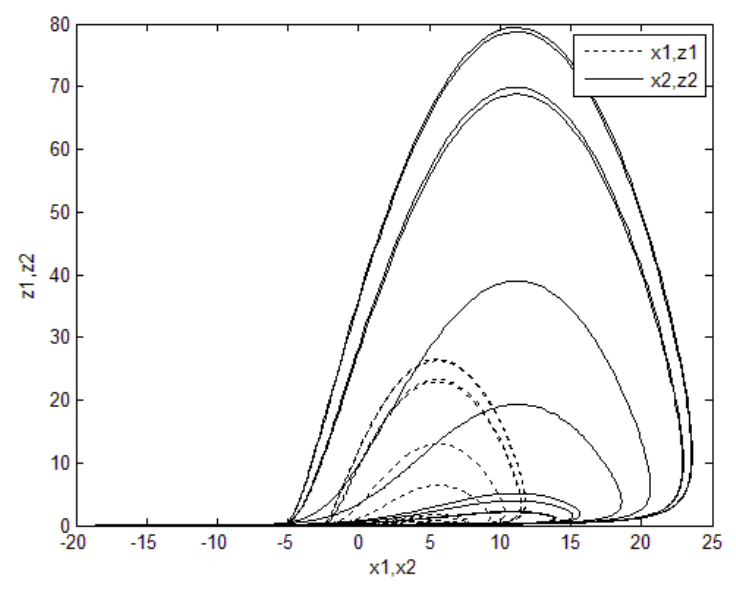

Figure 3. The attractors in projective synchronization for different systems.(3)

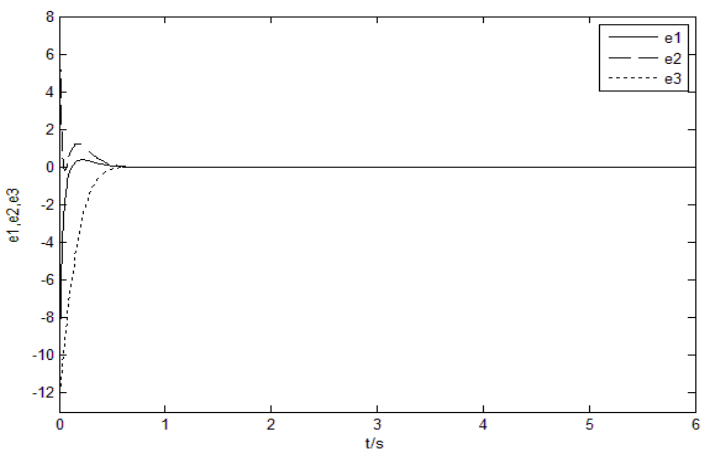

Figure 4. The synchronization errors between the different systems

Next, the simulation result for the projective synchronization between two identical chaotic systems is shown briefly. Where the system parameters is given by $a=0.2, b=0.2, c=5.7$. The initial values of the state variables for the two system are $(3,-5,3)$ and $(-4,5,-3)$. The scaling factors and the feedback gain coefficients are taken as $\alpha=1, \beta=\gamma=0.6, k_{1}=10, k_{2}=3, k_{3}=7$ respectively. Then the attractors in projective synchronization for the identical systems and the synchronization errors between the state variables are shown in figure 5 -figure 8. 


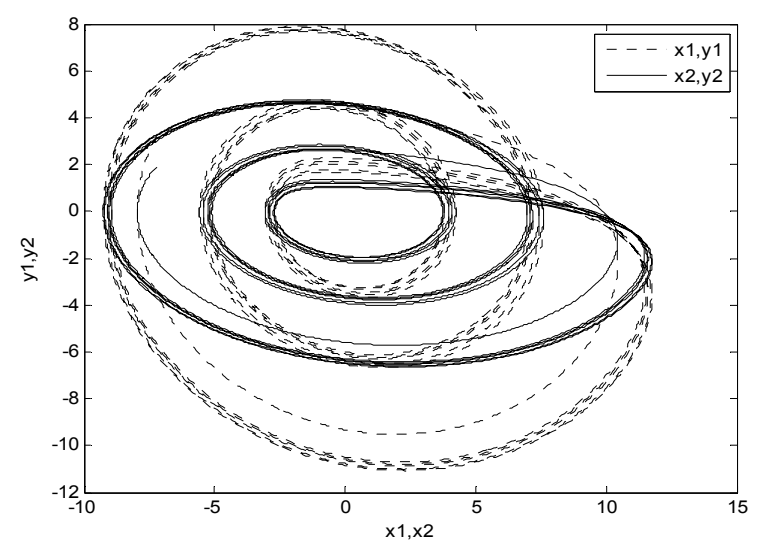

Figure 5. The attractors in projective synchronization for identical systems (1)

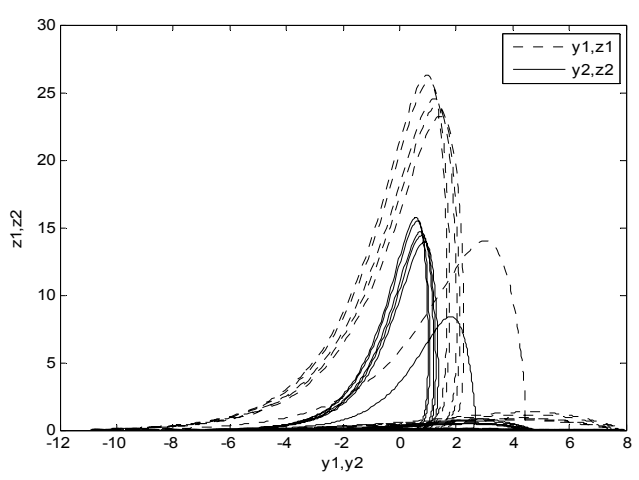

Figure 6. The attractors in projective synchronization for identical systems (2)

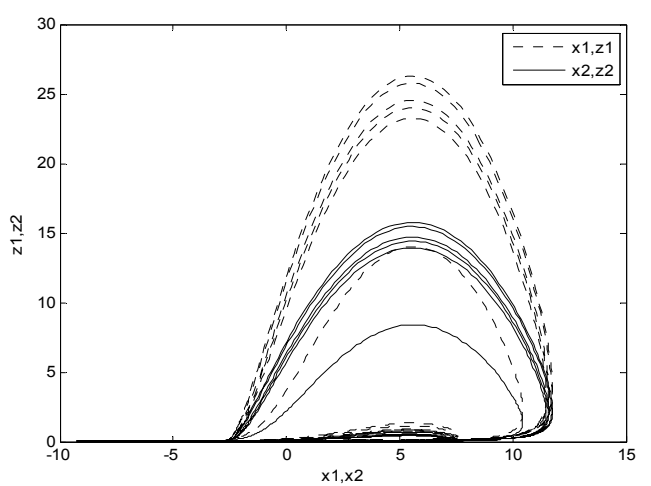

Figure 7. The attractors in projective synchronization for identical systems (3)

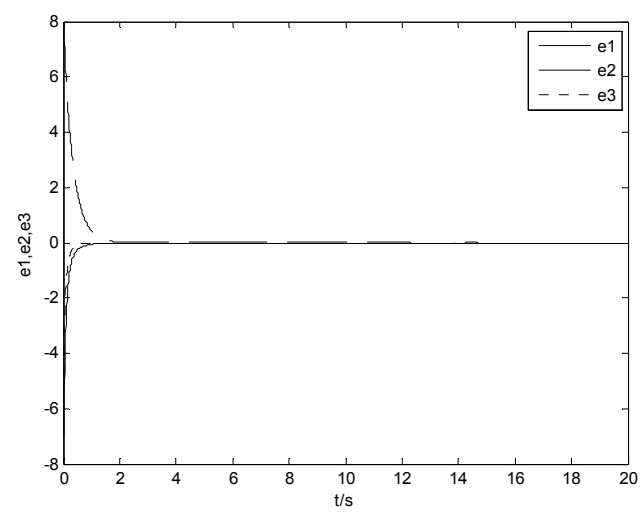

Figure 8 . The synchronization errors between the identical systems

\section{SUMMARIES}

This paper is concerned with the projective synchronization for different chaotic systems and identical systems via a nonlinear feedback control. The domain of the feedback gain coefficients which associated with the nonlinear controllers are computed by estimating the value of the state variables from the attractor of the drive system. In numerical simulations, the effectiveness and the feasibility of the proposed scheme have been demonstrated using Rössler and Lorenz chaotic systems. This method can be also applied to other chaotic system as a general approach.

\section{REFERENCES}

[1] Mainieri R; Rehacek J. Projective synchronization in threedimensional chaotic systems. Phys. Rev. Lett. [J], 1999.82, PP3042-5.

[2] LI Guo-hui. Generalized projective synchronization between Lorenz system and Chen's System. Chaos Solitons Fractals[J], 2007.32, PP1454-1458.

[3] LI Guo-hui. Modified projective synchronization of chaotic system. Chaos Solitons Fractals[J], 2007.32, PP1786-1790.

[4] LI Guo-hui. Modified projective synchronization of chaotic system. Chaos Solitons \& Fractals[J], 2007.32, PP1786-1790.

[5] Park Ju H. Adaptive modified projective synchronization of a unified chaotic system with an uncertain parameter. Chaos Solitons Fractals[J], 2007.34, PP1552-1559.

[6] Park Ju H. Adaptive controller design for modified projective synchronization of Genesio-Tesi chaotic system with uncertain parameters. Chaos Solitons Fractals[J], 2007.34, PP1154-1159.

[7] JIA Qiang. Projective synchronization of a new hyperchaotic Lorenz system. Physics Letters A[J], 2007.370, PP40-45.

[8] CHEN, Yong; LI, Xin. Function projective synchronization between two identical chaotic systems. Int. J. Mod. Phys. C[J], 2007.18, PP883-888.

[9] Park Ju H. Adaptive control for modified projective synchronization of a four-dimensional chaotic system with uncertain parameters. Comput. Appl.Math[J], 2008, PP:288-293.

[10] CHEN Yong; AN Hong-li; LI Zhi-bin. The function cascade synchronization approach with uncertain parameters or not for hyperchaotic systems. Comput. Appl. Math.[J], 2008.197, PP96-110.

[11] AN Hong-li; CHEN Yong. The function cascade synchronization method and applications. Commun. Nonlinear Sci. Numer. Simul.[J], 2008.13, PP2246-2255. 